\title{
Unnötige Regeln*
}

\author{
Elke Hentschel (Bern)
}

\begin{abstract}
:
Unnecessary rules

This paper argues that among the rules used in foreign language teaching there are often unnecessary ones. These rules offer detailed description of linguistic facts that probably could be learned easier without them. Although "explanatory rules" of this sort might be quite helpful when offered as additional explanations, they can hinder the language learning process when presented as rules to be learned and obeyed ("learning rules").

In order to show the difference between this set of rules more clearly, several examples are given. They belong to the context of German as a foreign language and concern the declension and comparison of the adjective, diminutives, and the passive voice.
\end{abstract}

\section{$1 \quad$ Lernregeln und Erklärungsregeln}

In der traditionellen Grammatikschreibung unterscheidet man zwischen präskriptiven und deskriptiven Ansätzen, also zwischen der Beschreibung der erwünschten, "korrrekten" Formen einer Sprache und solchen, die von den Nutzerinnen und Nutzern unter Missachtung vieler Vorschriften und ohne Rücksicht auf das ästhetische Empfinden von Sprachpuristen gebildet und eingesetzt werden. Die Übertragung dieser Unterscheidung auf den Fremdsprachenunterricht erscheint naturgemäß nur sehr beschränkt sinnvoll: wer eine fremde Sprache lernt, für den sind zunächst normalerweise alle Regeln präskriptiv in dem Sinne, dass es sich um Vorschriften handelt, die es zu beachten gilt. Wenn jemand nicht absichtlich ein bestimmtes, von der Norm abweichendes Register erlernen möchte, so gilt dies auch für die im eigentlich Sinne präskriptiven Regeln, bei denen man zusätzlich erfährt, dass sich die Muttersprachler über ihre Einhaltung nicht immer einig sind. Letzteres ist erfahrungsgemäß oft eher verwirrend als hilfreich, und Lernende stützen sich normalerweise auf die präskriptive Seite der Grammatik der jeweiligen Zielsprache, ohne - insbesondere in den frühen Phasen des Spracherwerbs - besonders viel Interesse an der Beschreibung von Abweichungen zu haben. Aber selbst wenn Lernende in Einzelfällen den Wunsch haben, eine "nicht korrekte" Varietät einer Fremdsprache zu erwerben, also etwa einen Dialekt, ein gruppenspezfisches Register o. ä., sind die Regeln dieser Varietät, auch wenn sie nicht der präskriptiven Grammatik folgen, für sie immer noch genauso bindend wie die der geltenden Norm, und in diesem Sinne abermals präskriptiv.

Dennoch soll die grundlegende Unterscheidung zwischen präskriptiv und deskriptiv, zwischen zu befolgender Vorschrift und einfacher Beschreibung des Vorhandenen, im Folgenden für den Bereich DaF fruchtbar gemacht werden - allerdings in einer leicht veränderten Be-

\footnotetext{
* Für ihre Ratschläge zu diesem Aufsatz danke ich ganz herzlich Theo Harden, Dublin, und Harald Weydt, Frankfurt (Oder). 
deutung und daher auch mit neuen Bezeichnungen. Als präskriptive Regeln, die es zu beachten gilt, sollen diejenigen Regeln betrachtet werden, die man beim Fremdsprachenerwerb auf die eine oder andere Art auswendig lernen muss. Beispiele hierfür sind etwa die Paradigmen, die Kongruenzregeln oder die Reihenfolge der verbalen Konstituenten in Haupt- und Nebensätzen. Um einen Spracherwerb zu ermöglichen, der die Lernenden nicht zu Pidgin-Sprechern macht, die dann von den Deutsch sprechenden Gesprächspartnern als ungebildete Angehörige der Unterschicht wahrgenommen und entsprechend behandelt werden, sind Regeln dieser Art unumgänglich. Sie sind im wahrsten Sinne des Wortes präskriptiv, denn sie schreiben vor, wie Deutsch als fremde Sprache richtig gebraucht wird. Da sie gelehrt und gelernt werden müssen, sollen sie im Folgenden als "Lernregeln" bezeichnet werden.

Nur beschreibende, in diesem Sinne also deskriptive Regeln wären im Kontext des Fremdsprachenunterrichts demgegenüber solche, die das Zusammenwirken verschiedener Elemente in der fremden Sprache erklären, es u.U. mit den Regeln der Muttersprache oder einer weiteren Fremdsprache vergleichen und den Regelapparat auf diese Art und Weise verständlicher und nachvollziehbarer machen. Sie beschreiben nur, sie schreiben nicht vor; so gesehen kann man auf sie verzichten. Damit sind sie jedoch nicht per se unnötig; ihr sinnvoller Einsatz ist möglich, er ist nur an andere Bedingungen geknüpft, als dies bei den Lernregeln der Fall ist.

Keiner der beiden Regeltypen ist also per se unnötig. Was sind dann "unnötige" Regeln? Es sind in der überwiegenden Mehrzahl der Fälle Erklärungsregeln, die sich sozusagen als Lernregeln verkleidet haben. Darüber hinaus können sich gelegentlich aber auch Lernregeln, die für bestimmte Gruppen von Lernenden relevant sind, sich bei anderen Gruppen als unnötig erweisen. Auf den sinnvollen oder eben unnötigen Einsatz dieser verschiedenen Regeltypen soll im Folgenden eingegangen werden.

\section{Sinnvoller Einsatz von Erklärungsregeln}

Zunächst soll der sinnvolle Einsatz von Erklärungsregeln an einem einfachen Beispiel verdeutlicht werden: an der Adjektivdeklination. Hier handelt es sich unbestreitbar um einen Bereich, der besonders lernaufwendig ist - der Formenbestand ist relativ groß, die Paradigmen sind umfangreich und scheinen erfahrungsgemäß vor allem zu Beginn des Lernprozesss unübersichtlich und abschreckend. Entsprechend versuchen moderne Lehrbücher, die Adjektivdeklination sozusagen häppchenweise einzuführen und den abschreckenden Gesamtüberblick möglichst lange hinauszuzögern. Dennoch kann es unter Umständen sinnvoll sein, die Paradigmen mit entsprechenden Beziehungssubstantiven (also so etwas wie: der schöne Mann, des schönen Mannes, dem schönen Mann... die kluge Frau, der klugen Frau... etc.) schlicht auswendig zu lernen. Das abrufbare Paradigma kann den Einsatz der richtigen Adjektivform erleichtern.

Das paradigma der Adjektivdeklination gehört natürlich in den Bereich der Lernregeln. Dem gegenüber enthält die Erklärungsregel zur Adjektivdeklination die Information, warum sich überhaupt ein derartiges Schema herausgebildet hat. Sie ist, wenn man so will, natürlich auch ihrerseits ein Stück weit präskriptiv, denn sie enthält die grundlegende, wenngleich abstrakte Regel, aus der alle Formen abgeleitet werden können. Eine solche Erklärung sucht man in vielen Grammatiken allerdings vergebens. So enthält beispielsweise die Duden-Grammatik, die sich immerhin mit dem Satz "Zugleich versteht sie [i.e. die Duden-Grammatik, E.H.] sich als ein praktisches Handbuch für das Unterrichten von Deutsch als Fremdsprache" (Duden 1998: 5) zu dem Ziel bekennt, im Bereich DaF einsetzbar zu sein, keine Erklärung des Prinzips, das sich in der umfassenden und detailgenauen Darstellung spiegelt (ibd.: 267-293). 
Auch in der dreibändigen Grammatik des Instituts für deutsche Sprache, die sich zwar nicht als DaF-Grammatik versteht, sich aber wünscht, dass "in den Köpfen der Leser (...) ein Bild von der deutschen Sprache [entsteht]" (Zifonun et al. 1997: 17), findet man eine Erklärung für die Zustände, die hier im Deutschen herrschen, bestenfalls zwischen den Zeilen und auch nur dann, wenn man bereits einige solide Vorkenntnisse mitbringt:

In der starken Flexion wird die spezifische Markierungsleistung eines Determinativs von der Adjektivform übernommen; die Formen entsprechen weitgehend denen des definiten Artikels. Damit kann - bei fehlendem Determinativ - zugleich die linke Phrasengrenze gekennzeichnet werden. Dafür, daß in der starken wie in der gemischten Flexion der Genitiv Singular Maskulinum/Neutrum nicht auf -(e)s endet, gibt es die Erklärung, daß der Genitiv schon beim Substantiv gut markiert ist. Die Nominativ- und Akkusativ-Singular-Formen der gemischten Flexion leisten die Abgrenzung der Genusformen, während die Numerusdifferenzierung ja schon bei der Determinativ- bzw. Substantivform gut markiert ist (...).

(Zifonun et al. 1997: 47)

Dagegen findet sich die gesuchte Erklärung für das scheinbare Wirrwarr der Adjektivflexion in der Grammatik von Helbig und Buscha (2001), die schreiben:

(..) ist die Deklination des Adjektivs variabel, und zwar abhängig vom (vorausgehenden) Artikelwort beim Substantiv. Diese Abhängigkeit besteht darin, dass die vollen Endungen, die die grammatischen Kategorien des Genus, Numerus und Kasus ausdrücken, stets nur einmal - entweder beim Artikelwort oder beim Adjektiv - erscheinen. Dieses für das Deutsche charakteristische Prinzip der Monoflexion äußert sich beim Adjektiv wie folgt: (...)

Helbig/Buscha (2001: 273 f., Hervorheb. i.O.)

Abgesehen davon, dass man den Sachverhalt für konkrete Unterrichtszwecke sicherlich etwas einfacher ausdrücken und darstellen würde, erhält man mit einer derartigen Erklärungsregel eine in der Tat außerordentlich hilfreiche Hintergrundinformation, die das System gut beschreibt. ${ }^{1}$

Auch wenn man die konkreten Formen damit noch nicht gelernt hat, kann man sie so in einen sinnvollen Kontext einbetten und durch logische Überlegungen bereits weitgehend ableite $n$, welche Form im konkreten Fall gebraucht wird.

\footnotetext{
1 In dieselbe Richtung weist auch die Erklärung bei Hentschel/Weydt ( ${ }^{2} 1994$ 189): "Die unterschiedlichen Endungen der Adjektive können damit erklärt werden, daß die vollständige Markierung von Kasus, Genus und Numerus jeweils nur einmal vorgenommen werden muß. Wenn sie bereits im Artikel ausgedrückt wird, muß das Adjektiv nicht mehr seinerseits sämtliche Informationen enthalten. Der gemischte Formenbestand nach dem unbestimmten Artikel kann damit darauf zurückgeführt werden, daß der unbestimmte Artikel in der Form ein gleichzeitig den Nominativ Maskulinum und Neutrum sowie den Akkusativ Neutrum ausdrückt; genau diese Fälle sind es, in denen die starke Deklinationsendung des Adjektivs verwendet wird."
} 
Die systematische Information zur Erleichterung des Verständnisses der Adjektivdeklination lässt sich noch erweitern. Wenn man zusätzlich weiß, dass das Deutsche den Akkusativ nur im Maskulinum Singular markiert - eine sehr grundlegende Regel, die sich nicht auf die Adjektivdeklination beschränkt, sondern das gesamte System einschließlich der Substantive und Pronomina umfasst - dann wird die bei Helbig/Buscha (ibd.: 274) folgende Aufzählung für die schwache Adjektivdeklination schon fast überflüssig:

\author{
Sing. Nom aller Genera \\ Sing. Akk.Neutr./Fem \\ alle anderen Kasus
}

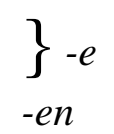

und dasselbe gilt für einen großen Teil der nachfolgenden Angaben zu den Endungen bei unbestimmtem Artikel und Nullartikel (ibd. 274 f.). Wenn man das grundlegende, umfassende Strukturprinzip kennt - das ja nicht nur die Adjektive, sondern alle Genus-flexiblen Wortformen betrifftt ${ }^{2}$ und das Eigenschaften wie beispielsweise die beinhaltet, dass der Akkusativ nur im Maskulinum Singular markiert wird - kann man die Adjektivdeklination sozusagen richtig erraten: man wird immer die korrekte Endung finden. Fehler, die man auf der Basis dieses Systems noch machen kann, entsprechen den Fehlern, die sich zunehmend bei deutschen Muttersprachlern beobachten lassen: die Regel, dass zwei Adjektive parallel zu flektieren sind, wird zugunsten des Prinzips "Einmal markieren genügt" aufgegeben. Daraus folgen dann Anredeformen wie Liebe Studierenden! oder Sehr geehrte Abgeordneten! Normativ gesehen sind sie falsch, denn bei zwei parallel gebrauchten Adjektiven oder Partizipen - die ja der Adjektivdeklinatuion folgen - müssen identische Endungen stehen. Aber systematische betrachtet sind sie sehr logisch, denn sie befolgen nur die Regel, dass einmaliges Ausdrücken von Kasus, Genus und Numerus genügt und man sich beim zweiten Mal mit der AllzweckEndung -en begnügen kann. (Ich wage sogar zu behaupten, dass es sich hier um Fehler handelt, sie man im Fremdsprachenunterricht fruchtbar machen kann - abgesehen von der sehr verständlichen Schadenfreude der Lernenden darüber, dass die Muttersprachler des Deutschen ihr vertracktes System selbst nicht richtig anwenden können, zeigt der Fehler so gut wie kaum ein anderes Beispiel, welche Regeln die Muttersprachler selbst zur Bildung der Formen anwenden.)

Dennoch wird interessanterweise in der Unterrichtspraxis eher selten auf solche explikativeren, strukturellen Regeln zurückgegriffen. Dies mag damit zusammenhängen, dass sie sich auf einer recht abstrakten Ebene der Sprachbeschreibung befinden, die von vielen in der DaFPraxis Tätigen gemieden wird. Mehrheitlich wird wohl befürchtet, dass die Lernenden damit überfordert würden - obwohl es Anlass zu der Annahme gibt, dass dieser Zugang, konsequent gewählt und in einer dem jeweiligen Niveau der Lernenden angemessenen Weise erklärt, umgekehrt als Wegweiser durch den Dschungel der Einzelregeln dienen und die Orientierung wesentlich erleichtern könnte. Eine mögliche Ursache für den Verzicht auf derartige Erklärungsregeln könnte in der didaktisch gut gemeinten Tendenz bestehen, Lernende im Zweifelsfall lieber zu unterfordern, als ihnen möglicherweise zu viel zuzumuten. Aber bei einer konsequenten Unterscheidung zwischen Lern- und Erklärungsregeln ergibt sich möglicherweise hier gar kein Konflikt mehr: die Erklärungsregel ist ein Angebot, das genutzt werden kann, aber nicht muss; die Lernregeln können dadurch vereinfacht und für viele leichter zugänglich gemacht werden. Der alternative Zugang über das Auswendiglernen des Paradigmas ist damit ja nicht verschlossen, sondern kann, wenn er als angenehmer empfunden wird, nach wie vor auch als alleiniger Weg gewählt werden.

\footnotetext{
${ }^{2}$ Zum Markierungssystem der gesamten nominalen Wortarten im Deutschen cf. auch Admoni ( ${ }^{4} 1982$ : 139f.).
} 


\section{Drei Beispiele für den Einsatz unterschiedlicher Regeltypen: Komparation, Diminution, Passiv}

Bisher war von einem Fall die Rede, in dem der Einsatz zusätzlicher Regeln sinnvoll war - also genau das Gegenteil von dem, was der Titel dieses Beitrages, "Überflüssige Regeln", erwarten lässt. Im Folgenden soll nun am Beispiel von drei anderen Fällen aus verschiedenen Bereichen der Morphologie (incl. Morphosyntax) gezeigt werden, wie Regeln aussehen, die als überflüssig angesehen werden können. Diese drei Beispielfälle sind die Komparation, die Diminution und das Passiv.

\subsection{Komparation des Adjektivs}

Obwohl moderne wissenschaftliche Grammatiken vereinzelt dazu tendieren, die Komparation zur Wortbildung und nicht zur Flexion zu rechnen (cf. Zifonun et al. 1997: 47f.), wird sie mehrheitlich letzterer zugerechnet (cf. zur Diskussion auch Eisenberg 1998: 176f., Hentschel/Weydt ${ }^{2}$ 1994: 189). Wo auch immer man sie ansiedelt: in den Lehrbüchern und Grammatiken für DaF darf sie im Gegensatz zu Wortbildungsmustern, auf deren Einübung zum aktiven Gebrauch aus gutem Grund oft verzichtet wird, nicht fehlen.

Als Beispiel für eine mögliche Darstellung soll hier zunächst wiederum die Duden-Grammatik angeführt werden. Dabei geht es nun nicht um die Regeln für die Bildung der konkreten Formen von Komparativ und Superlativ, sondern um die Ausnahmen: konkret um die Erläuterung, bei welchen Adjektiven eine Komparation nicht möglich ist. Die Duden-Grammatik (1998: 306f.) schreibt hierzu:

Bei bestimmten Adjektiven ist es in der Regel nicht möglich, Vergleichsformen zu bilden, weil bei ihnen auf Grund ihrer Bedeutung im allgemeinen kein Vergleich und keine Gradabschattung möglich isr. Folgende Gruppen sind hier anzuführen:

1. Adjektive, mit denen bestimmte Verfahren oder Zustände ausgedrückt werden, die einen Vergleich

verschiedener Grade ausschließen:

schriftlich, mündlich, wörtlich, ledig, sterblich, viereckig, rund, tot, lebendig, leblos,

stumm, nackt usw.

Hierher gehören auch zusammengesetzte Adjektive, deren Bestimmungswort bereits eine Verstärkung bezeichnet: schneeweiß, blutjung, steinreich, urkomisch, riesengroß, altklug usw.

Adjektive wie maximal, minimal, total, absolut, erstklassig, mit denen bereits ein höchster oder geringster Grad ausgedrückt wird, werden trotzdem gelegentlich (z. B. in der Werbesprache) gesteigert, um den Ausdruck des höchsten bzw. geringsten Grades möglichst noch zu verstärken (minimalster Verschleiß, erstklassigste Ausführung).

Möglich sind Vergleichsformen auch von Adjektiven, die an sich einen höchsten bzw. geringsten Grad ausdrücken, daneben aber auch in relativer Bedeutung verwendet werden können: Was leer ist, kann an sich nicht leerer sein, was still ist, nicht am stillsten. Gebraucht der Sprecher/Schreiber diese Adjektive aber nicht in ihrer absoluten, sondern in einer relativen Bedeutung, dann kann er auch vergleichen:

Das Kino ist heute leerer als gestern. In den stillsten Stunden der Nacht... Geschichten müssen vergangen sein, und je vergangener, könnte man sagen, desto besser für sie (Th. Mann). Hier aber zeigte sich Frau Stöhrs große Unbildung im vollsten Licht (ders.). ... es ist der vollkommenste Aufbau, den man sich denken kann (Koeppen).

2. Adjektive, mit denen das im Stammwort Ausgedrückte verneint wird: unrettbar, unüberhörbar, unverlierbar usw.

Verschiedentlich sind jedoch auch hier Vergleichsformen möglich: Er ist noch unordentlicher als du. Selbst die unempfindlichsten Menschen

3. Adjektive, mit denen das Fehlen des im Stammwort Genannten ausgedrückt wird: kinderlos, bargeldlos, obdachlos, fleischlos. 
Vergleichsformen sind aber auch hier möglich bei solchen Adjektiven, die weniger konkreten Inhalt haben:

... die fruchtloseste Diskussion. Eine zwanglosere Zusammenkunft war nicht vorstellbar. Lieblosere Briefe gab es wohl nicht.

4. Zahladektive (vgl. aber 514,2): letzt, einzig', neunfach, ganz, halb usw.

5. Indeklinable Farbadjektive (vgl. 445,5): oliv, rosa, lila.

6. Von Adjektiven, die nur attributiv (vgl. 450) oder nur prädikativ (vgl. 45 1) gebraucht werden, sind im Allgemeinen Vergleichsformen unüblich, es sei denn - und das gilt auch für die unter 1-5 besprochenen

Adjektive -, sie werden in übertragener Bedeutung verwendet: eine lebendigere Darstellung... Die Straße ist lebloser als gestern. Er arbeitet mit eisernstem Fleiß.

Vergleichsformen werden auch gelegentlich dann gebildet, wenn Adjektive, mit denen an sich nur die Herkunft charakterisiert wird (vgl. 450,2), als Artadjektive gebraucht werden:

Er ist der schwäbischste unter diesen Dichtern. Gleich sah sie französischer aus (Baum).

Bei Adjektiven, die ursprünglich Substantive gewesen sind (vgl. 451), können Gradunterschiede nur durch Umschreibungen ausgedrückt werden.

7. Von den Partizipien, die wie ein Adjektiv gebraucht werden (vgl. 333), werden vor allem dann Vergleichsformen gebildet, wenn sie innerhalb der Wortart Verb isoliert sind (vgl. 335):

(Nur:) das schreiende Kind, der ausgesprochene Tadel; (gegenüber [isoliert]:) das reizendste Geschenk, der gelehrteste Vortrag, in schreiendsten Farben. (Aber auch:) der gefürchtetste Meeresbewohner.

Bei den anderen Partizipien werden die Gradunterschiede zumeist durch Umschreibungen ausgedrückt, wenn die Bedeutung des Verbs dies zuläßt:

der mich am meisten verdrießende Umstand, der mehr bietende Käufer, das am meisten besprochene Problem, das meistgelesene Blatt.

Der Text mit dieser Erklärung umfasst fast zwei eng bedruckte Seiten; er ist sicher ein Extrembeispiel, belegt aber gut, wie weit man hier ins Detail gehen kann.

Welchen Erklärungswert haben die hier aufgestellten Regeln?

Komparation als semantisches Prinzip - wenn auch keineswegs notwendigerweise als morphologisch oder morphosyntaktisch am Adjektiv realisiertes Phänomen - kann mit Sicherheit in allen Sprachen der Welt auf irgend eine Art ausgedrückt werden. Dies ist schon allein daraus zu folgern, dass die kognitive Fähigkeit, Eigenschaften im Hinblick auf ihre Intensität miteinander zu vergleichen, zumindest im Bereich der räumlichen Dimension, allen Menschen gegeben sind: Wir können unterscheiden, ob ein Objekt größer oder kleiner als ein anderes, ein Weg länger oder kürzer, ein Ort näher oder weiter entfernt ist etc. Langacker (2000: 2; 94; 361) zählt die kognitive Fähigkeit des Vergleichens zu den Basisfähigkeiten, die für das Denken wie die Sprache gleichermaßen grundlegend sind. Sein Begriff der "Comparison" impliziert dabei natürlich sehr viel mehr, als für die grammatische Komparation von groß-größer oder tief-tiefer erforderlich ist; aber auch diese Art des sprachlichen Vergleichs kann aus der grundlegenden Fähigkeit hergeleitet und somit als universale kognitive Dimension angesehen werden.

Der Unterschied zwischen den einzelnen Sprachen liegt damit nicht in ihrer Möglichkeit, solche Vergleiche grundsätzlich in irgend einer Weise zum Ausdruck zu bringen, sondern nur darin, welche sprachlichen Mittel sie hierzu nutzen. Dabei lassen sich mit Bezug auf das Ad- 
jektiv grundsätzlich zwei Prinzipien unterscheiden, die hier am Beispiel der Komparativbildung aufgezeigt werden sollen: ${ }^{3}$

- morphologische Komparation (freie oder gebundene Morpheme):

(1) synthetisch, cf. Deutsch, Englisch: schöner [als]; nicer [than])

(2) analytisch, cf. Französisch, Englisch: plus beau [que]; more beautiful [than])

- syntaktische Komparation:

(1) mit fakultativem freiem Morphemen, cf. Türkisch: [Ablativ] ${ }^{4}\left(\right.$ daha $\left.^{5}\right)$ güzel 'schön'

(2) ohne zusätzliche Morpheme, cf. Mongolisch: [Ablativ] гоё 'schön'

Diese unterschiedlichen Bildungsweisen müssen natürlich beim Erwerb der Sprache mit gelernt werden. Was aber mit Sicherheit nicht gelernt werden muss, ist die Regel, dass man normalerweise von Wörtern wie tot, hölzern, chinesisch oder viereckig keinen Komparativ bildet. Die Sprache, in der so etwas zum alltäglichen Sprachgebrauch gehören würde, lässt sich schwer vorstellen. Weltwissen und allgemeine kognitive Fähigkeiten reichen hier voll und ganz aus, um Formen wie töter, chinesischer etc. gar nicht erst entstehen zu lassen. Dasselbe gilt aber auch umgekehrt für die Ausnahmen von dieser Regel: wenn man ausdrücken möchte, dass eine Eigenschaft, die normalerweise nur vorliegt oder eben nicht vorliegt, aber keine Abstufungen zulässt, in einem besonderen Fall doch in stärkerem Maße vorhanden ist, als man es unter normalen Umständen erwarten würde, dann ist auch die ad-hoc Komparation solcher Adjektive sowohl möglich als auch zulässig. Ein Adjektiv wie gleich schließt selbstverständlich Steigerung aus; aber wenn uns Orwell von der Farm der Tiere erzählt, haben wir weder mit dem englischen Gebot des Schweins Napoleon: "All Animals Are Equal, But Some Animals Are More Equal Than Others" noch mit seiner deutschen Übersetzung - die zwar je nach Text für some einige oder manche benutzt, für others andere oder andere Tiere schreibt, aber stets einen Komparativ von gleich bildet - ein Problem grammatischer oder anderer Art. Sowohl die Komparation dieses Adjektivs als auch die Ironie dieser Bildung sind unmittelbar zugänglich.

Nichts von alledem muss gelernt werden. Es kann zwar interessant sein, den Sachverhalt zu beschreiben - im Sinne einer oben definierten Erklärungsregel - aber der Hinweis darauf, dass die Beschränkungen semantischer Natur sind, sollte deutlich als hilfreiche Information kenntlich gemacht werden. Auf keinen Fall handelt es sich dabei um eine Regel, die man zusätzlich lernen muss, und schon gar nicht sollte sie mit einer Liste von Adjektiven verknüpft werden, die es auswendig zu lernen gilt. Wenn sie so präsentiert wird, liegt der Fall einer Erklärungsregel im Gewand einer Lernregel vor - und in dieser Form ist die Regel dann mit Sicherheit wenig hilfreich und unnötig, möglicherweise sogar schädlich. Denn Schaden kann eine solche überflüssige Regel insofern anrichten, als sie den Zugang zum intuitiven Sprachwissen und -verständnis verbaut, indem ein einfach zu lernender Sachverhalt hinter einem Gestrüpp von Regeln und Ausnahmen verschwindet und damit unzugänglich wird.

\footnotetext{
${ }^{3}$ Stassen (1985) unterscheidet dem gegenüber auf der Grundlage der Gesamtkonstruktion, jedoch ohne besondere Berücksichtigung des morphologischen Verhaltens des Adjektivs, sechs resp. vier Typen: drei mit Lokativ-Konstruktionen (unter Verwendung dreier verschiedener lokaler Kasus, die zu den semantischen Bereichen 'woher', 'wo' und 'wohin' gehören), einen mit einem 'übertreffen'-Verb ('A ist über B hinausgehend schön'), den kombinierenden Konstruktionstyp 'A ist schön, B ist nicht schön' und den Partikel-Komparativ 'A ist schöner als B'.

${ }^{4}$ Der Hinweis auf den Ablativ an derselben Stelle, wo oben deutsch als, französisch que, englisch than als Beispiele angeführt werden, bedeutet, dass das Vergleichsobjekt in diesen Sprachen mit einem Ablativ angeschlossen wird. Ein Satz wie İstanbuldan güzel, wörtlich: 'Istanbul-ABL schön', wäre somit etwa als 'schöner als Istanbul' wiederzugeben.

${ }^{5}$ Daha lässt sich etwa mit 'noch' übersetzen: daha güzel 'noch schöner'.
} 


\subsection{Diminutivbildung}

Die Diminutivbildung, die interessanterweise in den drei Bänden der IdS-Grammatik kein einziges Mal erscheint, bildet gewissermaßen das substantivische Gegenstück zur Komparation, wobei auch die Richtung, in der die Veränderung der Bedeutung geht, genau entgegengesetzt ist: einem Mehr beim Adjektiv steht ein Weniger beim Substantiv gegenüber. ${ }^{6}$ Mehrheitlich wird sie - im Unterschied zur Komparation - zur Wortbildung gerechnet (cf. DudenGrammatik 1998: 504). Dies führt nun normalerweise dazu, dass weniger Regeln und Vorgaben für die aktive Bildung solcher Formen gegeben werden, da Wortbildung als notorisch unregelmäßig und entsprechend schwer aktiv zu erlernen angesehen wird. Im Falle der Diminutiva ist dies jedoch nicht so, und man findet gewöhnlich Anweisungen zur Bildung der sog. Verkleinerungsformen. Hier kann man zwei Arten von Hinweisen unterscheiden: morphologische und semantische. Während es selbstverständlich sehr sinnvoll ist, darauf hingewiesen zu werden, dass das Vorliegen eines Suffixes wie -ling oder einer Pluralendung den Gebrauch der Diminutivendung verhindert (cf. *Schmetterlingchen, *Hühnerchen; Ausnahmen wie Kinderlein bestätigen die Regel), ist die Aufzählung von semantischen Einschränkungen hier so wenig sinnvoll wie bei der Komparation der Adjektive. Sprecher von Sprachen, in denen ebenfalls Diminutiva gebildet werden können, kennen das semantische Konzept. Sie wissen z. B. intuitiv, dass es unter normalen Umständen nicht angebracht ist, von Giftschlänglein und Kanalrättchen zu reden. Die so bezeichneten Tiere mögen zwar klein sein; niedlich, harmlos oder liebenswert sind sie jedoch normalerweise nicht. Das kann sich jedoch ändern, wenn ein Märchen von der Familie Giftschlange (mit Mama Schlange, Papa Schlange und dem kleinen Schlängelein) oder der ebenso netten Familie der Kanalratten erzählt wird; dann sind die Diminutiva aber auch wieder möglich. Bei abstrakten Begriffen wie Liebe, Hass oder Zorn schließt eben die Tatsache, dass es sich um abstrakte Konzepte handelt, für alle, die Diminutiva als Konzeption bereits kennen, Bildungen wie *Häßchen oder *Zörnlein aus, und wenn die Form wie in Liebchen doch vorkommt, wird sie automatisch konkret und bezeichnet die geliebte Person, nicht das abstrakte Gefühl. Sprechern von Sprachen, in denen es keine Diminutiva gibt, muss in erster Linie dieses grundlegende semantische Prinzip vermittelt werden; ${ }^{7}$ wenn sie wissen, dass sie mit dem Diminutiv nicht einfach nur 'geringe Größe' ausdrückt, sondern zugleich eine bestimmte, positive emotionale Haltung zum so beschriebenen konkreten Objekt transportiert wird, können sie die Formen richtig verstehen und auch einsetzen, ohne dass sie zusätzlich Einzelregeln brauchen.

Das wirkliche Problem liegt hier auf der pragmatischen Ebene: wann es angemessen ist, ein Diminutiv zu gebrauchen, deren Frequenz ja nicht nur von Sprache zu Sprache (man denke nur an die vielen im Deutschen recht unmotiviert anmutenden Onkelchen, die in Übersetzungen russischer Texte zu finden sind), sondern auch innerhalb des Deutschen regional erheblich variiert. Dies aber ist wiederum eine ganz andere Art von Regel als diejenigen, von denen bisher die Rede war.

\footnotetext{
${ }^{6}$ Das Mehr bei Substantiv, also die Bildung von Augmentantiva, existiert selbstverständlich auch und ist in vielen Sprachen, so etwa dem Italienisch oder dem Serbischen, voll ausgebildet; im Deutschen jedoch gibt es bestenfalls rudimentäre Ansätze zu solchen Bildungen, wie sie etwa in Unmenge, Unsumme oder Untiefe (in der Bedeutung 'große Tiefe') vorliegen.

${ }^{7}$ Man könnte die These vertreten, dass es sich beim zugrundeliegenden Prinzip um ein universelles, eher kognitives als sprachliches Muster handelt; wenn dies der Fall ist, würde das zugleich bedeuten, dass die Vermittlung besonders einfach wäre.
} 


\subsection{Passiv}

Als drittes Beispiel sollen nun die Regeln und Einschränkungen zur Passivbildung betrachtet werden. Hier handelt es sich um ein komplexes Gebiet aus dem Bereich der Verbalmorphologie und Morphosyntax, das in der Mehrzahl der Lehrbücher erst nach der Komparation, aber weit vor der Diminutivbildung, behandelt wird (cf. z.B. Mebus et al. 1987). Zentral für das richtige Verständnis und den korrekten Gebrauch von Verbformen, ist das Passiv zugleich eine gefürchtete Quelle für Fehler wie auch für Erklärungsprobleme. Dabei kann man besonders gut beobachten, welche Regeln sinnvoll sind, welche ergänzend sinnvoll sein können und welche sich als überflüssig, manchmal sogar eher hinderlich für den Spracherwerb erweisen.

$\mathrm{Zu}$ den sinnlosesten Regeln, die mir in meiner eigenen DaF-Praxis begegnet sind, gehört mit Sicherheit die folgende: "Das persönliche Passiv kann man nur von transitiven Verben bilden". Sie fand sich auf einem Merkblatt, das Studierenden am Studienkolleg der TU Berlin ausgehändigt worden war; einer von ihnen hatte sich damit hilfesuchend an mich gewandt und bat mich, ihm diese Regel zu erklären. Natürlich konnte ich das nicht: sie ist tautologisch. ${ }^{8}$ Was sie zu transportieren versuchte, ist indes leicht zu erraten: es war der Hinweis darauf, dass man von sog. pseudotransitiven Verben wie haben, bekommen etc. oder von unpersönlichen Konstruktionen wie es gibt trotz des Vorhandenseins eines Akkusativs im Aktiv kein Passiv bilden kann. Dieser Hinweis findet sich regelmäßig in der einen oder anderen Form, manchmal auch recht ausführlich, in den Grammatiken (cf. Duden 1998: 179, Engel 1996: 453, Helbig/Buscha 2001: 153, Hentschel/Weydt ${ }^{2}$ 1994: 119, Zifonun et al. 1997: 1796-1799). Die interessanteste Frage lautet hier: wie wichtig ist diese Regel? Werden Deutschlernende ohne sie in Versuchung kommen, Sätze mit unmöglichen Passivformen wie *Das Buch wird (von mir) gehabt, *Zum Geburtstag wurde von mir ein Buch bekommen oder *Im Herbst wird Obst gegeben zu bilden?

Vielleicht werden sie solche Formen bilden, wenn der Fremdsprachenunterricht mehrheitlich auf dem Lernen der verschiedensten Regeln basiert, die dann mechanisch angewendet werden sollen, auch wenn man sie nicht recht versteht - aber sie würden spontan und von sich aus vermutlich kaum versuchen, so etwas zu sagen. In Nominativsprachen wie dem Deutschen ist die Rolle des Agens im unmarkierten Standardfall (also im Falle eines Handlungsverbs im Aktiv) mit dem Subjekt verknüpft, und zugleich ist das Vorliegen eines Agens im Subjekt auch stets die Voraussetzung dafür, dass ein Passiv möglich ist. ${ }^{9}$ Gerade die einzige Verbform, bei der man das Agens getrost weglassen darf, setzt kompromisslos das Vorliegen eines Agens voraus. Und dies ist zugleich die übergeordnete, sprachübergreifende Regel für die Passivbildung; sie gilt unabhängig davon, welche konkreten Konstruktionen in der einzelnen Sprache möglich sind, und schließt nicht nur pseudotransitive Verben aus, sondern gibt darüber hinaus auch die Bedingung an, unter der ein intransitives Verb ein Passiv bilden kann. Man kann sich somit fragen: Kommt wirklich jemand von sich aus auf die Idee, in der zu lernenden Sprache einen Satz wie *Von der Wurst wird geschimmelt oder *Im Park wird von den Rosen geduftet zu bilden, so dass er durch Verblisten und Regeln von diesem Vorhaben abgehalten werden muss? Oder kann man nicht mit einiger Wahrscheinlichkeit annehmen,

\footnotetext{
${ }^{8}$ Dies gilt dann, wenn man den traditionellen Transitivitätsbegriff verwendet, der Passivierbarkeit zur Bedingung macht (cf. hierzu z.B. Glück 2000: 745 und die dort angegebe Literatur); in derselben Bedeutung wird der Begriff auch in der modernen kognitiven Grammatik gebraucht (cf. z.B. Langacker 2000: 210). Dem gegenüber steht ein gelegentlich zu findender erweiterter Transitivitätsbegriff, in dem die Grenzen verwischt und auch Verben mit indirekten Objekten eingeschlossen werden (so beispielsweise bei Bußmann 1990: 806); wendet man ihn an, dann ist die Regel sogar falsch.

${ }^{9}$ Damit soll nicht behauptet werden, dass alle Sprachen dieses Typs über ein Passiv verfügen; möglicherweise ist es sogar nur eine Minderheit (cf. Haspelmath 1990).
} 
dass diese Bildungen etwa so häufig vorkommen wie der Versuch, die Rosen mit einem freundlichen Bittet duftet! zur Absonderung von Geruch aufzufordern oder die Wurst mit Bitte schimmle nicht! um längere Haltbarkeit zu bitten? Natürlich kann man all dies tun - und wenn man es tut, kann man Rosen und Wurst auch mit Passivformen wie Hier wird nicht geschimmelt! oder Jetzt wird aber endlich mal kräftig geduftet! ermahnen, um den Erfolg dieser Aufforderung dann befriedigt mit Daraufhin wurde von allen Rosen brav geduftet zu konstatieren. Das Problem ist, wie diese Beispiele zeigen, abermals nicht wirklich grammatischer Natur, sondern hängt davon $\mathrm{ab}$, ob man beim Gebrauch eines Verbs intentionales Handeln und damit ein Agens voraussetzt.

Es kann angenommen werden, dass diese Regel den Lernenden in der Mehrheit (nämlich dann, wenn ihre Muttersprache zu den Nominativsprachen gehört) zwar nicht bewusst ist, dass sie ihnen aber zumindest auf derselben Ebene zugänglich ist, auf der auch die Regeln ihrer Muttersprache verankert sind. Wenn man sich auf diese sprachliche Intuition verlässt, oder genauer: wenn man die Lernenden dazu veranlasst, sie zu nutzen, bedarf es keiner komplizierten Regeln und Wörterlisten, aus denen dann entnommen werden kann, welche Verben nicht passivfähig sind. Man kann die Agentivität als Voraussetzung für jegliche Passivbildung als zusätzliche Erklärungsregel anbieten und sich ansonsten anderen Schwierigkeiten zuwenden.

Diese anderen Schwierigkeiten sind gerade beim Passiv natürlich immer vorhanden, können aber je nach Muttersprache stark variieren und einen entsprechend unterschiedlichen Einsatz von Regeln verlangen. Typische Stolpersteine sind beispielsweise der Unterschied zwischen dem werden- und dem sehr viel selteneren sein-Passiv oder der Ausschluss eines Dativ-Passivs (im Sinne eines Transfers des Dativ-Objekts in die Subjektposition des Passivsatzes, also eine Form wie $* D u$ wirst/bist geholfen) bei diesen beiden Auxiliaren. Der Ausschluss solcher Formen gehört natürlich zur normativen Ebene der deutschen Grammatik und ist insofern eine Lernregel. Aber hier kann man versuchen, die Regeln der jeweiligen Muttersprache fruchtbar zu machen: wenn diese kein Dativpassiv zulässt, ist es höchst unwahrscheinlich, dass es zur spontanen Bildung dieser Form beim Erwerb des Deutschen kommt, und die Lernenden müssen nicht mit einer weiteren Regel konfrontiert werden, die ihnen etwas verbietet, was sie ohnehin nicht tun wollen. Dies wäre ein Beispiel für den oben erwähnten Fall einer unnötigen Lernregel. Wenn die Muttersprache der Lernenden vergleichbare Konstruktionen zulässt, ist hier natürlich die Einführung dieser Lernregel unabdingbar.

Das eigentliche Rezipientenpassiv, das mit den Verben bekommen oder kriegen gebildet werden kann, spielt seltsamerweise sowohl in den Grammatiken als auch im Bereich DaF nur eine sehr untergeordnete Rolle, obgleich es sehr viel frequenter ist als beispielsweise das seinPassiv, das stets behandelt wird (cf. Hentschel/Weydt 1995: 178-182). Eine weitere Überlegung im Rahmen des hier vorgenommenen Versuches, Regeln zu werten, könnte daher natürlich auch dahin gehen, zu berücksichtigen, wie frequent eine Form sein sollte, damit sie zu welchem Zeitpunkt des Spracherwerbs auch zum aktiven Gebrauch vermittelt werden muss (und nur für diese Vermittlung des aktiven Gebrauchs sind Lernregeln nötig). Muss man ein sein-Passiv bilden können oder reicht es, solche Formen zu verstehen und möglicherweise gelegentlich zu spiegeln? Es wäre zu überlegen, ob hier nicht Erklärungen analoger Art ausreichen, wie sie für Wortbildungsphänomene gegeben werden. Wie bereits erwähnt wird die Wortbildung, beispielsweise die Derivation von Substantiven auf -heit und -keit, mehrheitlich nicht aktiv erlernt, sondern nur für das passive Verständnis vermittelt. Dabei kann man die Lernenden ermutigen, bereits gehörte Formen ruhig auch zu verwenden, aber nicht selbst neue zu bilden. Vielleicht wäre dies gerade für Fälle wie das sein-Passiv, über dessen Bil- 
dungsbedingungen sich die Grammatiken des Deutschen ja alles andere als einig sind ${ }^{10}$ eine sehr praktikable Lösung. Man kann eine einfache Erklärungsregel zur formalen Bildung und Bedeutung dieser Formen anbieten und ansonsten wahrheitsgemäß darauf hinweisen, dass sich die Grammatikschreibung nicht ganz einig ist, unter welchen Bedingungen und von welchen Verben sie gebildet werden können. Damit erspart man den Lernenden eine Vielzahl von komplexen und uneinheitlichen Lernregeln und ermöglicht es ihnen dennoch, mit den Formen umzugehen.

\section{$4 \quad$ Fazit}

Die Anzahl der Regeln, die als "Lernregeln" in den Unterricht eingeführt werden müssen, ist mit Sicherheit sehr viel kleiner als diejenige der in den meisten Fällen eingeführten und gelernten Regeln. Dies kann unter Umständen dazu führen, dass die Lernenden keinerlei Intuition für die zu lernende Sprache entwickeln, sondern sich mit einem System von unübersichtlichen und wenig einsichtigen Regeln konfrontiert sehen, die zu lernen und zu befolgen sind. Das Lernen überflüssiger Regeln schadet nicht nur dem Lernerfolg und produziert unnötige Fehler, sondern beschädigt auch das Ansehen der zu lernenden Sprache, die dann als schwer erlernbar gilt und immer weniger Interessenten findet. Gerade das Deutsche scheint, möglicherweise auf Grund der großen Zahl der sprachintern konkurrierenden Grammatiken und Sprachbeschreibungsmodelle, in besonderem Maße unter diesem Phänomen zu leiden.

Aus diesem Grund ist es sinnvoll, präskriptive "Lernregeln" von deskriptiven Erklärungsregeln zu unterscheiden, die nur zu beschreiben versuchen, was in der Mehrheit der Fälle intuitiv zugänglich ist. Wenn man den letzteren Regeltyp im Unterricht einführt, sollte er als solcher kenntlich gemacht werden. Wenn irgend möglich, sollte dabei stets auch die muttersprachliche Kompetenz und möglicherweise darüber hinaus auch allgemeine kognitive Fähigkeiten der Lernenden fruchtbar gemacht werden. Dabei können die Lernenden insbesondere in Einrichtungen der höheren Bildung wie Gymnasien und Universitäten auch angeregt werden, solche Regeln anhand von muttersprachlichem wie fremdsprachlichem Material selbst zu finden. Ein "Das ist ja genauso wie bei uns!" hilft naturgemäß sehr beim Bau einer Brücke zur anderen Sprache, und das Bewusstsein, dass die Zahl der wirklich fremden, neu zu lernenden Regeln nicht wirklich groß ist, tut ein Übriges.

\section{Literatur:}

Admoni, Waldimir ( $\left.{ }^{4} 1984\right)$ : Der deutsche Sprachbau. 4., überarbeitete und erweiterte Auflage. München: Beck.

Bußmann, Hadumod (1990): Lexikon der Sprachwissenschaft. Zweite, völlig neu bearbeitete Auflage. Stuttgart: Kröner.

Duden (1998), Grammatik der deutschen Gegenwartssprache. 6., neu bearb. Auflage, herausgegeben von der Dudenredaktion, bearbeitet von Peter Eisenberg u. a. Mannheim: Dudenverlag.

Eisenberg, Peter (1998): Grundriss der deutschen Grammatik. Das Wort. Stuttgart/Weimar: Metzler.

\footnotetext{
${ }^{10}$ Helbig/Buscha (2001: 156f.) gehen von einer resultativen Bedeutung der Form aus, wobei das Resultat von Dauer (mit dem Verb bleiben verträglich) sein soll, so dass auch nur Verben mit "transformativer bzw. resultativer Bedeutung" (ibd.: 156) diese Form bilden können. Demgegenüber spricht der Duden (1998: 184) nur von "(oft schwer fassbaren) Abstufungen der Art, dass das Zustandspassiv bestimmter Verben durchaus üblich, das anderer Verben hingegen weniger oder gar nicht üblich ist", während die IdS-Grammtik "sein- und werdenPassiv in der Regel aspektuell differenziert" sieht (Zifonun et al. 1997: 1808).
} 
Glück, Helmut (ed.) (2000): Metzler Lexikon Sprache. Zweite, überarbeitete und erweiterte Auflage. Stuttgart/Weimar: Metzler.

Haspelmath, Martin (1990): "The grammaticalization of passive morphology." Studies in Language 14.1: 25-71.

Helbig, G./Buscha, J. (2001): Deutsche Grammatik. Ein Handbuch für den Ausländerunterricht. Neubarbeitung. Berlin etc.: Langenscheidt.

Hentschel, Elke/Weydt, Harald ( $\left.{ }^{2} 1994\right):$ Handbuch der deutschen Grammatik. Berlin/New York: de Gruyter.

Hentschel, Elke/Weydt, Harald (1995): "Das leidige bekommen-Passiv" In: Popp, Heidrun (ed.): Deutsch als Fremdsprache. An den Quellen eines Faches. Festschrift für Georg Helbig zum 65. Geburtstag. München, Iudicium: 165-183.

Langacker, Ronald W. (2000): Grammar and Conceptualization. Berlin/New York: Mouton de Gruyter.

Mebus, Gudula, et al. (1987): Sprachbrücke 1. Stuttgart: Klett.

Stassen, Leon (1995): Comparision and universal grammar. Oxford: Blackwell.

Zifonun, Gisela, et al. (1997): Grammatik der deutschen Sprache. 3 Bde. Berlin/New York: de Gruyter. 